\title{
PEDAGOGÍA DEL CUERPO SENSIBLE: TACTO Y VISIÓN EN LA DANZA CONTACT IMPROVISATION
}

\author{
PEDAGOGIA DO CORPO SENSÍVEL: TATO E VISÃO NA DANÇA CONTATO \\ IMPROVISAÇÃO
}

PEDAGOGY OF THE SENSITIVE BODY: TOUCH AND SIGHT IN CONTACT IMPROVISATION DANCE

\author{
Maria Paz Brozas Polo*
}

Palabras clave:

Percepción.

Contacto.

Danza.

Educación.

Palavras chave: Percepção.

Contato.

Dança.

Educação.

\begin{abstract}
Resumen: El objetivo de este artículo es analizar el lugar del tacto y de la visión en el marco de la pedagogía del cuerpo sensible en la danza Contact Improvisation $(\mathrm{Cl})$ a través de los contenidos de la sección didáctica de la revista Contact Quarterly. Nos basamos en las aportaciones de la filosofía y de la psicología de la percepción: aplicamos los conceptos de sinestesia de Merleau Ponty, así como los de tacto activo y visión kinestésica de Gibson. El estudio permite identificar conceptos, funciones y técnicas del entrenamiento sensorial en la praxis del $\mathrm{Cl}$ así como destacar las capacidades táctil y visual como dimensiones que no pertenecen solo a la mano 0 al ojo, respectivamente, sino a la totalidad del cuerpo.
\end{abstract}

Resumo: 0 objetivo deste artigo é analisar o lugar do tato e da visão no âmbito da pedagogia do corpo sensível na dança Contato Improvisação $(\mathrm{Cl})$ a partir dos conteúdos da seção educacional da revista Contact Quarterly. Com base nas contribuições da filosofia e da psicologia da percepção, nós aplicamos os conceitos de sinestesia de Merleau Ponty, bem como o do toque ativo e visão cinestésica de Gibson. 0 estudo permite identificar conceitos, funções e técnicas do treino sensorial da dança $\mathrm{Cl}$ e destacar as capacidades tácteis e visuais como dimensões que não pertencem apenas ao olho ou à mão, respectivamente, mas a todo o corpo.

Abstract: The aim of this article is to analyze the place of touch and sight within the pedagogy of the sensitive body in Contact Improvisation dance using contents of the journal Contact Quarterly's educational section. We rely on the contributions of the philosophy and psychology of perception, with Merleau Ponty's concepts of synesthesia and Gibson's views on active touch and kinesthetic sight. The study allows identifying concepts, functions and techniques of sensory training applied to this dance form as well as highlighting tactile and visual capacities as dimensions that do not belong only to hands or eyes respectively, but involve the whole body as well.
*Universidad de León. León, España. E-mail:mpbrop@unileon.es

Recebido em: 01-02-2017 Aprovado em: 27-07-2017 


\section{INTRODUCCIÓN}

El coreógrafo Steve Paxton, junto a un grupo de bailarines, planteó en el Nueva York de los años 70 una serie de experimentos coreográficos que fueron el germen de la técnica perceptiva de danza que denominó Contact Improvisation $(\mathrm{Cl})$. Hoy se reconocen sus aportaciones a la creación coreográfica contemporánea (BANES, 1987; BUCKWALTER, 2011; GIL, 2001; FONTAINE, 2004; LOUPPE, 2004; SUQUET, 2006), así como a la pedagogía de la danza (BALES; NETTL-FIOL, 2008; BENJAMIN, 2002; BRUGAROLAS, 2015'; COOPER ALBRIGHT, 2013; LEITE, 2005) y, en general, a la pedagogía corporal, con una particular atención en el ámbito de la educación física (BROZAS, 2000, 2013, 2016a, 2016b; BORQUE, 20152; TORRENTS; CASTAÑER, 2008; TORRENTS et al., 2010).

Desde los inicios del $\mathrm{Cl}$, como estudio práctico sobre el movimiento, se cuestionan las posibilidades de la relación física -y social- entre los cuerpos mediante del juego con el peso priorizando la atención a las sensaciones internas antes que al diseño de formas visibles de danza (PAXTON, 1975, 1993; PAXTON; BIGÉ, 2015). De este modo, el Cl propone una desviación tanto del paradigma del sentido de la vista en la sociedad moderna, como de la primacía de la imagen visual tan característica de la danza escénica occidental (PEETERS, 2014; TAMPINI, 2012).

Con estos antecedentes, en este trabajo se propone una revisión de los conceptos y de las funciones de los sentidos, en particular del tacto y de la vista, así como de la relación entre ellos, en el marco pedagógico de esta forma de danza. Mediante el procedimiento de cotejo documental y análisis de contenido se pretende extraer las referencias que, al respecto, aparecen en aquellos textos cuya intención es de índole didáctica; asimismo, se quiere poner de relieve el sustento teórico que, bien de carácter filosófico o bien de carácter psicológico, parece acompañar sus formulaciones. Nos apoyamos para ello en la ontología del cuerpo sensible de Merleau Ponty $(1964,1975)$, todo un nudo generador del discurso contemporáneo sobre el cuerpo expresivo y la danza (BERNARD, 1977, 2001), así como en la ecología de la percepción de James Gibson $(1962,2015)$, algunos de cuyos textos sirvieron de inspiración a Steve Paxton y, sobre todo, a la coreógrafa Lisa Nelson (2001), quien se ha dedicado al estudio de la visión en el movimiento improvisado.

La aproximación didáctica de Paxton al tacto y a los sentidos en la danza, fruto también de las investigaciones con personas con problemas o ausencia de visión (PAXTON; KILCOYNE; MOUNT, 1993), se completa con las de otros profesores expertos en Cl cuyas reflexiones se documentan en la revista Contact Quarterly, y de forma específica, en la sección pedagógica denominada Essentials. Se cuenta, finalmente, con la experiencia de esta autora como profesora y estudiosa de $\mathrm{Cl}$, desde finales de los años noventa, interesada, asimismo, en los modos de percepción particulares de los bailarines con y sin visión (BROZAS, 2016a).

\section{PEDAGOGÍA DE LA DANZA CI: PARADIGMA DE CUERPO SENSIBLE}

La atención que prestan a los sentidos los movimientos de renovación pedagógica, desde Rousseau hasta las Escuelas Nuevas pasando por Spencer (LUZURIAGA, 1923), se ve

1 BRUGAROLAS, Maria Luisa. El cuerpo plural. Danza integrada en la inclusión, una renovación de la mirada. Tesis doctoral inédita. Universidad de Valencia: Departamento de Escultura, 2015. 
respaldada por las investigaciones sobre el tono, la afectividad y la comunicación que se hicieran desde la psicobiología y la psiquiatría (VAYER, 1977). Surgen los tratados específicos sobre el tacto (ACKERMAN, 1992; MONTAGU, 1971), en conversación con las teorías de aprendizaje y desarrollo motor (USKI, 2003). Asimismo, en la segunda mitad del siglo XX se consolida la denominada corriente "corporeista" en la que la exaltación del cuerpo se ve sustentada en las aportaciones de la sociología crítica, de la psicología humanista y de la fenomenología existencialista a fin de cuestionar el código social imperante y de reivindicar el disfrute sensorial (LIDELL, 1989; PICARD, 1983). Mientras tanto, la danza moderna y, más aún, la danza posmoderna se cuestionan los modos de percepción y transmisión del movimiento a la vez que exploran el lugar de los sentidos en los procesos de creación coreográfica (BROZAS 2013).

El paradigma de cuerpo sensible en la danza $\mathrm{Cl}$, se sitúa, por tanto, en un amplio marco de referencias, constituyéndose como un crisol permeable que se nutre tanto de técnicas de conciencia corporal como de postulados filosóficos, psicológicos y pedagógicos. A este respecto, Suquet $(2006$, p. 381, 396) define el Cl como una forma de diálogo ponderal que coloca el sentido del tacto en el centro de la danza y lo considera uno de los estilos de danza que ha reinventado de manera más profunda la esfera perceptiva. Por su parte, Tampini (2012) analiza el uso del tacto y de los sentidos en el $\mathrm{Cl}$ como un desplazamiento respecto del modus operandi de la sociedad moderna, donde la prevalencia absoluta de la visión como ojo escrutador y cortical exige del entendimiento una activación que desactiva, a su vez, la sensibilidad hacia el entorno y hacia los otros cuerpos. El $\mathrm{Cl}$, junto a otras formas de danza contemporánea, participa de lo que Desprès (2001) denomina una "pedagogía de la emergencia" en la que el profesor quiere dejar de ser modelo para convertirse en inductor de marcos, estructuras y juegos; para ello, el cuerpo aprende la experimentación autónoma con el entorno y con los otros cuerpos a través de los sentidos. El cuerpo sensible del $\mathrm{Cl}$, más que el cuerpo recto y gracioso que aún prevalece en la danza escénica, aspira a la disolución del ego en el paisaje físico y social. Este cuerpo sensible en relación consigo mismo, con los demás cuerpos danzantes y con el entorno, se constituye, por tanto, como referente no solo de una pedagogía de los sentidos sino de una pedagogía de la inclusión, así como de una pedagogía "ecosomática", basada en la diversidad y en la reciprocidad (CLAVEL; GINOT, 2015).

\title{
3 EL TACTO Y LOS SENTIDOS EN LA DIDÁCTICA DEL CI
}

En uno de sus primeros artículos sobre $\mathrm{Cl}$, Steve Paxton define el $\mathrm{Cl}$ como un sistema dual basado en los sentidos del tacto y del equilibrio:

\begin{abstract}
Los miembros del dúo se tocan mucho uno a otro pues es a través del tacto como se transmite la información sobre el movimiento del otro. Además, tocan el suelo, y ahí está el énfasis en la constante conciencia de la gravedad. También están en contacto consigo mismos internamente y se mantiene una concentración en la totalidad del cuerpo (PAXTON, 1975, p. 40). [Traducción propia]
\end{abstract}

Algunos años más tarde, en Drafting interior techniques, Paxton (1993) señala la importancia del entrenamiento específico de los sentidos y propone desarrollar el hábito de la observación en la posición de pie -con el ejercicio que denominó Small Dance-pero también en el curso de los movimientos más extremos -cuando el movimiento es demasiado rápido para el pensamiento y las acciones reflejas adoptan el rol principal- y cuando se pierde la referencia de la horizontalidad y la continuidad visual; también se pierde la estabilidad del tacto porque 
el suelo deja de ser la superficie estable de apoyo al utilizar otras superficies corporales en movimiento en las que se basa la danza. Según la observación de mi propia práctica, durante la relativa quietud que proporciona la posición vertical, leyendo el suelo con los pies y leyendo al mismo tiempo los movimientos que se dibujan en el suelo a través de las plantas, se genera un amplio espacio para experimentar con las interferencias y las acciones complementarias de tocar y mirar; cuando los movimientos son más rápidos y cuando implican cambios de nivel, orientación y emplazamiento, emerge también la necesidad de focalizar los sentidos dejando jugar a la visión periférica con las sensaciones inmediatas del tacto, configurando el uso de una especie de "tacto periférico".

Aprender a bailar $\mathrm{Cl}$, según Paxton (1993), implica perder inhibiciones y tabúes relacionados con el contacto, explorar nuevos movimientos corporales y variaciones de las sensaciones periféricas y alcanzar un estado estable que permita observar y sentir los reflejos. Para ello, cada uno de los sentidos proporciona imágenes de diferente naturaleza, pero las más necesarias en el trabajo del $\mathrm{Cl}$ son las imágenes kinestésicas y las herramientas específicas que Paxton denomina las técnicas interiores.

Estas técnicas se refieren al uso de un tacto interno y profundo que viaja a través de la piel, pero, además, a través de los músculos y de los huesos, y que nos permite percibir todo el cuerpo, pero además el otro cuerpo y sus sutiles movimientos: por ejemplo, a través del punto de contacto en "cabeza con cabeza", cada bailarín puede sentir la pequeña danza de su compañero. Se trata de una observación y experimentación de la mente-movimiento del otro (PAXTON, 1996, p. 50).

Con el fin de conocer el lugar que ocupan el tacto y la visión en los procesos de enseñanza de la danza $\mathrm{Cl}$ en los últimos años, se ha consultado la sección pedagógica de Contact Quarterly denominada Essentials. Se han revisado todas las propuestas, desde el primer año en que aparecen, 2006, hasta 2016. Dicha sección incluye principios fundamentales, ejercicios y reflexiones procedentes de diferentes profesores expertos en la enseñanza del $\mathrm{Cl}$.

Para un mejor cotejo de las propuestas didácticas se ha elaborado un cuadro a cuatro columnas (Cuadro 1). En la primera se recogen los datos de los autores. En la segunda se enuncian los títulos; respecto de estos es preciso señalar que, aunque no contengan referencias explícitas ni al tacto ni a la vista, el contenido está más o menos directamente orientado al uso y desarrollo del tacto. En la tercera columna se recogen las consignas más representativas de cada propuesta; aunque ninguna se refiere al sentido de la vista de forma independiente, dos de ellas (BROOK, 2016; LEPKOFF, 2008), incorporan las alusiones a la vista como parte del desarrollo del tacto. Finalmente, en la cuarta columna, se propone un listado de variaciones 0 categorías táctiles, derivadas del contenido de cada uno de los artículos.

Cuadro 1- Referencias al tacto y la visión en la sección pedagógica Essentials.

\begin{tabular}{|c|c|c|c|}
\hline $\begin{array}{c}\text { Autores y años } \\
\text { Ray Chung } \\
(2006)\end{array}$ & Títulos & Consignas sobre tacto y visión & ¿Qué tacto/visión? \\
\hline $\begin{array}{c}\text { Karen Nelson } \\
(2006)\end{array}$ & $\begin{array}{c}\text { Tones of } \\
\text { Relaxation }\end{array}$ & $\begin{array}{c}\text { De la relajación a la extensión usando } \\
\text { el compañero como superficie y } \\
\text { ofreciendo superficie }\end{array}$ & Tacto profundo \\
\hline
\end{tabular}


Continuación del cuadro $1 . .$.

\begin{tabular}{|c|c|c|c|}
\hline $\begin{array}{l}\text { Nita Little } \\
\text { (2007) }\end{array}$ & Place and Pour & $\begin{array}{c}\text { Desde el contacto con la piel dirige el } \\
\text { peso a través de la estructura ósea } \\
\text { hacia el suelo. }\end{array}$ & Tacto vacío o lleno (peso) \\
\hline $\begin{array}{l}\text { Martin Keogh } \\
\quad(2007)\end{array}$ & Falling & $\begin{array}{l}\text { Leyendo el suelo a través de los pies } \\
\text { del compañero. }\end{array}$ & Tacto extendido, prolongado \\
\hline $\begin{array}{l}\text { Daniel Lepkoff } \\
\qquad(2008)\end{array}$ & $\begin{array}{l}\text { An Ordinary } \\
\text { Movement }\end{array}$ & $\begin{array}{l}\text { Modulando la mano, modulando todo } \\
\text { el cuerpo: el contacto ha de ser firme, } \\
\text { pero no demasiado duro. }\end{array}$ & $\begin{array}{c}\text { Tacto ocular, manual y } \\
\text { vertebral }\end{array}$ \\
\hline $\begin{array}{l}\text { Cris Aiken } \\
(2008)\end{array}$ & Moulding /Draping & $\begin{array}{c}\text { Usa un tacto ligero: lo mínimo para } \\
\text { sentir y escuchar activamente } \\
\text { (Alexander Technique). Toca sin dirigir. }\end{array}$ & Tacto adaptable \\
\hline $\begin{array}{l}\text { Martin Hugues } \\
\text { (2009) }\end{array}$ & $\begin{array}{l}\text { Dance with the } \\
\text { body You Have }\end{array}$ & $\begin{array}{l}\text { Crea un pequeño gesto (...) Repite tu } \\
\text { gesto. ¿Están tus dos pies en el suelo? }\end{array}$ & Tacto múltiple \\
\hline $\begin{array}{l}\text { Alito Alessi } \\
\text { (2009) }\end{array}$ & $\begin{array}{l}\text { Yes and Know } \\
\text { Work }\end{array}$ & $\begin{array}{l}\text { Tacto, comunicación e imaginación: } \\
\text { cualquier parte del cuerpo y diferentes } \\
\text { formas de contacto. }\end{array}$ & Tacto diverso y abierto \\
\hline $\begin{array}{l}\text { Nina Martin } \\
(2010)\end{array}$ & The Fussy Dance & Movimiento y pausas de un segundo. & Tacto veloz e intermitente \\
\hline $\begin{array}{l}\text { Charlie Morrissey } \\
\text { (2011) }\end{array}$ & Room to move & $\begin{array}{l}\text { La piel contenedora, el cuerpo en } \\
\text { expansión, las manos respirando. } \\
\text { Ofreciendo espacio y resistencia en el } \\
\text { contacto. }\end{array}$ & Tacto poroso y resistente \\
\hline $\begin{array}{l}\text { K.J. Holmes } \\
\text { (2012) }\end{array}$ & $\begin{array}{l}\text { You walk into a } \\
\text { room }\end{array}$ & $\begin{array}{l}\text { Piel y respiración: océano de } \\
\text { sensaciones, otros cuerpos como } \\
\text { paisajes. Tocando el espacio. }\end{array}$ & Tacto espacial \\
\hline $\begin{array}{c}\text { Neige } \\
\text { Christenson } \\
\text { (2013) }\end{array}$ & $\begin{array}{l}\text { Modulation } \\
\text { of Tone and } \\
\text { Connection to } \\
\text { Center }\end{array}$ & $\begin{array}{l}\text { Cierta cantidad de tono permite crear } \\
\text { una clara conexión, un circuito para } \\
\text { compartir el peso. }\end{array}$ & Tacto enérgico \\
\hline $\begin{array}{l}\text { Kristin Horrigan } \\
\text { (2014) }\end{array}$ & Slow-Motion Duet & $\begin{array}{l}\text { Respira, notando la sensación, } \\
\text { presiona el punto de contacto y espera } \\
\text { para sentir y moverte con curiosidad. }\end{array}$ & Tacto lento e intenso \\
\hline $\begin{array}{l}\text { Margaret Sunghe } \\
\text { Paek } \\
(2015)\end{array}$ & $\begin{array}{l}\text { Beginning, } \\
\text { Approaching, } \\
\text { Practicing, } \\
\text { Dancing }\end{array}$ & Toma una pareja, cambia de pareja. & Tacto disponible \\
\hline $\begin{array}{c}\text { Annie Brook } \\
\text { (2016) }\end{array}$ & $\begin{array}{l}\text { Awaking the back } \\
\text { Space }\end{array}$ & $\begin{array}{l}\text { Las percepciones se afinan hacia el } \\
\text { espacio posterior al movernos. Con } \\
\text { atención e imaginación. Body Mind } \\
\text { Centering. }\end{array}$ & $\begin{array}{l}\text { Tacto interno } \\
\text { Vista dorsal }\end{array}$ \\
\hline
\end{tabular}

Fuente: Elaboración propia a partir de la revista Contact Quarterly. Sección Essentials (2006-2016).

En la primera de las referencias de la sección pedagógica, Ray Chung (2006, p. 316) nos da la clave para alcanzar un estado de comunicación profunda gracias a la habilidad de la escucha: "[...] la práctica de recibir información de otra persona en un nivel físico a través del toque". Escuchar y tocar significan esperar, seguir, ceder y atestiguar, la propuesta resalta el compromiso activo de escuchar a través del tacto, así como su particular importancia durante el proceso de liderazgo del movimiento.

Los quince artículos pedagógicos revisados en Contact Quarterly (Cuadro 1) nos permiten subrayar algunas funciones específicas del tacto según los diferentes autores; 
en primer lugar, escuchar y leer la forma, la dinámica y la dirección en el propio cuerpo, en otros cuerpos y en el espacio circundante (CHUNG, 2006; KEOGH, 2007; LITTLE, 2007); en segundo lugar, probar distintas posibilidades de movimiento (ALESSI, 2009; HUGUES, 2009); y finalmente, acompañar con atención los movimientos de los otros cuerpos (KEOGH, 2007; SUNGHE PAEK, 2015). Esta tercera idea, la de cuidar a los otros durante la danza a través del sentido del tacto la desarrolla Annie Brook (2016), cuyo método nos insta, sobre todo, a leer el espacio desde la espalda, desafiando la restricción impuesta por la frontalidad de nuestra vista.

Estas propuestas didácticas nos ofrecen evidencias sobre la relevancia de otros elementos con los que se relacionan íntimamente los sentidos durante el aprendizaje como son el tono (CHRISTENSON, 2013; LEPKOFF, 2008; NELSON, 2006), la respiración (HOLMES, 2012; HORRINGAN, 2014; MORRISSEY, 2011), el peso (CHUNG, 2006; LITTLE, 2007) o la velocidad (HORRIGAN, 2014; MARTIN, 2010). Por su parte, aparecen referencias explícitas a otras técnicas corporales como la Técnica Alexander (AlKEN, 2008) y el Body Mind Center (BROOK, 2016), cuyas aportaciones al $\mathrm{Cl}$ remiten a dos formas particulares de tacto: en la primera, se propone un tacto no directivo, preciso y sensible (ROBIN, 2008) y, en la segunda, un tacto también interno, que no se alberga únicamente en la piel más externa sino en las membranas de todos los órganos (BAINBRIDGE-COHEN, 1993).

\section{APROXIMACIÓN ONTOLÓGICA AL TACTO-VISIÓN EN LA DANZA CI: RECIPROCIDAD Y SINESTESIA}

Una de las características primordiales del sentido del tacto es la reciprocidad: cuando estamos tocando a alguien, al mismo tiempo, y necesariamente, estamos siendo tocados por ese alguien. Esta reciprocidad del tacto, menos evidente en el caso de la vista, puede ser analizada desde diversos puntos de vista en el marco pedagógico del $\mathrm{Cl}$.

Durante el contacto, cuando el cuerpo que está tocando alcanza otro cuerpo -persona o cosa-, le transmite su propia información al tiempo que recibe información de lo que está tocando a través de la superficie del cuerpo que toca. Por otra parte, al tocar otro cuerpo, el que toca recibe información sobre sí mismo. "Cuando te estoy tocando, ¿te toco a ti o a mí misma? Puedo centrarme en percibirme utilizando tu cuerpo como mi entorno o puedo centrarme en ti, en la percepción de tu textura y tu forma." (NELSON, 2008, p. 104). Esta divergencia entre el interior y el exterior, como dos focos posibles de la experiencia, constituye "el natal secreto del cuerpo", de lo que Merleau Ponty denominó nuestro cuerpo sensible (MERLEAU PONTY, 1964, p.135).

Al mismo tiempo, cuando un bailarín toca a otro bailarín, el doble proceso de percepción tiene lugar en los dos bailarines: saber que uno está tocando y siendo tocado está unido a la conciencia de que el mismo proceso está siendo experimentado por la persona con la que estamos bailando (PAXTON, 1996). Para José Gil (2001), la reciprocidad en el Cl implica un incremento de energía corporal; esta energía se transmite a través del tacto de forma inconsciente, pero hay conciencia del proceso de transferencia y es la conciencia del contacto lo que permite la comunicación de los dos inconscientes. De este modo, tiene lugar una doble fusión: entre el cuerpo y la conciencia y entre los dos cuerpos; de ahí emerge la idea de un único cuerpo colectivo con dos singularidades.

Por otra parte, en el $\mathrm{Cl}$, los conceptos de tocar y ser tocado resultan inseparables debido a los cambios permanentes de rol entre el mover y el ser movido, pues al bailar es 
necesario un equilibrio entre proponer y seguir al otro. Al respecto, Lisa Nelson explica cómo el liderazgo se desplaza constantemente no solo desde quien está moviendo y tocando a quien está siendo movido y tocado, sino también entre los distintos sentidos que intervienen al bailar. Según su planteamiento, nuestro cuerpo es un instrumento de afinación compuesto por sutiles antenas diferenciadas: nuestros sentidos. De este modo, "[...] estamos siempre nadando y bailando en señales que recibimos del entorno a través de nuestros ojos y oídos y a través del tacto; [...] con el tacto, sin visión, aprender un movimiento de alguien nos lleva mucho tiempo, el espacio se acerca y la imaginación se incorpora" (NELSON, 2001, p.23); esta perspectiva nos sugiere la idea del apoyo mutuo de los sentidos durante el aprendizaje.

Una aproximación más radical entre visión y tacto se encuentra en los escritos de Merleau Ponty (1964), quien justifica la reciprocidad de la visión por la sinergia entre estos dos sentidos: para él, la visión se describe como una palpación con la mirada, de un mismo cuerpo viendo y tocando en una sola acción conjunta. Además, nos propone la extensión de la reciprocidad característica del tacto a la vista:

\begin{abstract}
El que mira está también en el mundo que está mirando: el que mira no puede poseer lo visible a menos que sea también poseído por ello [...] Hay intrusión, encabalgamiento, no solo entre lo tocado y lo tocante, sino también entre lo tangible y lo visible, que se incrustan uno en otro, y a la inversa; lo tangible en sí mismo no es nada sin la visibilidad, no existe sin la experiencia visual (MERLEAU PONTY, 1964, p. 134). [Traducción propia]
\end{abstract}

En todo caso, de acuerdo con Gibson (1962, p. 477), el tacto activo, referido a la acción de tocar, ha de distinguirse del tacto pasivo, referido a la acción de ser tocado porque el tacto activo es un sentido exploratorio y no meramente receptivo. El tacto activo implica sentirse en contacto, pero también sentirse en movimiento: al tocar (con las manos) se produce un escaneo táctil similar al ocular, derivado del movimiento de los dedos, en el que la variación en la estimulación de la piel procede de su actividad motora. Mientras el tacto receptivo funciona desde la sensibilidad cutánea, el tacto activo se relaciona no solamente con la piel y su tejido subyacente, sino también con los músculos y, sobre todo, con los receptores de las articulaciones y los tendones; se puede considerar, por ello, "un sentido articular". El sentido del tacto implica el juego de impulsos que llegan desde la totalidad del sistema esqueléticomuscular: no hay un simple órgano o estructura como un oído o un ojo.

La experiencia nos indica que mientras se baila $\mathrm{Cl}$ el tacto se puede considerar siempre un tacto activo en un sentido aún más amplio que el definido por Gibson: el tacto receptivo (pasivo) y el exploratorio (activo) funcionan juntos desde que comienza el contacto en la danza. Este tacto activo en $\mathrm{Cl}$ significa, por tanto, sentirse en movimiento, pero puede significar también compartir el peso. El peso, incluso más que el movimiento, otorga la posibilidad de adquirir, con la práctica, un "tacto profundo" (Cuadro 1).

El desarrollo de un tacto activo-profundo implica, además, la recepción y la gestión de un abanico de sensaciones táctiles simultáneas o sucesivas basadas en diferentes focos que emergen en el flujo de movimiento: tocar el suelo y el aire al mismo tiempo, tocar con algunas partes del propio cuerpo diferentes partes de otros cuerpos. Esta es la razón por la que la danza Cl exige la adquisición de una especie de "tacto múltiple" (Cuadro 1); de la evidencia sobre múltiples focos de contacto se deriva a su vez la dificultad para definir un órgano del tacto. 
Mientras Gibson (1962) o Gaos (2010) refieren el sentido del tacto a la mano, en Cl se cuestiona este uso. Algunos profesores limitan o desaconsejan el uso de las manos durante el aprendizaje, hasta el punto de que se puede observar una especie de "manos muertas o colgantes" en algunos bailarines de $\mathrm{Cl}$. A principios de los ochenta una de las consignas características de la enseñanza del $\mathrm{Cl}$ era "evitar el contacto directivo de las manos" (BANES, 1987, p. 114). La cuestión sería determinar cuál puede ser es el lugar de las manos en una danza en la que, de todos modos, estas reciben el peso del cuerpo pues, como dice Louppe (2004, p. 66) el apoyo se reparte en el conjunto del cuerpo y no solo en el pie.

Sin embargo, es preciso reconocer cierta preponderancia en el papel sensitivo de las manos en varias de las propuestas revisadas en el apartado anterior (Cuadro 1), donde el uso del tacto se plantea, desde la mano hacia todo el cuerpo:

Grandes y lectoras manos reposan en el cuerpo de tu pareja. Tus manos son pasajeras del cuerpo -no se implican ni preguntan nada sino simplemente notan el feedback desde la periferia hasta el centro-. Codos y hombros permanecen blandos mientras notas alguna sensación de tu centro afectado por tu pareja mientras dejas tus manos en su cuerpo. Tus manos dejan espacio para que tu pareja se expanda. Las manos respiran (MORRISSEY, 2011, p. 37). [Traducción propia]

En la propuesta del profesor Lepkoff (2008, p. 53), se observa una particular referencia a los dedos: "Prepara los dedos para el primer contacto [...]. Tus dedos encuentran la superficie del vaso de papel. Tu mano se ciñe alrededor del vaso, y empiezas a aplicar una suave presión".

Gaos, desde la filosofía, (2010) señala la mano no solo como la parte hábil del cuerpo sino como la parte educada y sensible, la parte distanciada del suelo durante la evolución humana en el momento en que empezamos a caminar y a apoyarnos únicamente sobre los pies: la mano sería la parte cualificada para tocar y acariciar debido a su forma cóncava. Esta forma permite su extensión y su adaptación al otro cuerpo; permite un tacto muy suave que conecta piel y corazón. El tacto con la palma de la mano es, para este autor, un tipo de contacto que evoluciona hacia el tacto más distal del que se ocuparán los ojos.

A este respecto, según el análisis de Gibson (1976), habría una ligera extensión de las posibilidades táctiles de la mano a los miembros, pero sin dejar de considerar las manos y los ojos como las partes de nuestro cuerpo con la mayor movilidad y sensibilidad, es decir, los "órganos" de los sentidos que sostienen el tacto y la vista en nuestra vida cotidiana. No obstante, en su aproximación ecológica a la percepción visual, concibe cada uno de los órganos especializados como parte de un funcionamiento corporal global.

Uno ve el ambiente no solo con los ojos, sino con los ojos en la cabeza sobre los hombros de un cuerpo que se acerca. Miramos los detalles con los ojos, pero también miramos alrededor con la cabeza móvil, y avanzamos y miramos con el cuerpo móvil (GIBSON, 2015 p.211). [Traducción propia]

Desde esta perspectiva, la visión también es kinestésica en la medida en que registra los movimientos del cuerpo: tanto los de la totalidad del mismo con relación al soporte donde camina, como los movimientos parciales de las extremidades relativos al conjunto del cuerpo. Así, no solo se refiere al ojo como órgano de la visión, sino que otorga capital importancia tanto al giro de la cabeza que permite el barrido del campo visual, como a las variaciones de los miembros que redefinen las formas visibles del mismo y finalmente, al flujo de movimiento e imágenes procedente del desplazamiento (GIBSON, 2015). 
El tacto y la visión no tienen nada en común cuando se conciben como canales de datos sensoriales puros y sin significación, sin embargo, como canales para la recogida de información, con sentido activo y exploratorio, tienen mucho en común y ambos sentidos están normalmente activos, trabajando juntos (GIBSON, 1962). Por ejemplo, el horizonte se puede visualizar y se puede sentir en la medida en que cualquier superficie que se toca es experimentada con relación al plano horizontal (GIBSON, 1986, p. 124); la visión puede considerarse kinestésica en cuanto que registra movimientos del cuerpo tanto como el sistema músculo-esquelético-articular y el sistema vestibular: todos los sentidos obtienen información respecto del entorno y respecto del propio cuerpo cuando se consideran sistemas perceptivos (GIBSON, 1986).

Del mismo modo, la sinergia entre los sentidos, como señala Merleau Ponty, puede ayudarnos a entender la posibilidad de la sinergia entre diferentes cuerpos: “¿Por qué no podría existir la sinergia entre diferentes organismos si es posible dentro de ellos? Sus paisajes se entrelazan, sus acciones y sus pasiones encajan exactamente: esto es posible en el momento en el que pertenecemos a la misma consciencia" (MERLEAU PONTY, 1964, p. 142). La reciprocidad entre los sentidos parece ser la clave y el principio fundamental para alcanzar la comunicación entre los cuerpos:

Por primera vez deja el cuerpo de acoplarse al mundo, se abraza a otro cuerpo, aplicándose meticulosamente a él con toda su extensión (...). Y a partir de entonces, movimiento, tacto y visión, aplicándose al otro y a sí mismos, ascienden hacia su origen y, con la acción paciente y silenciosa del deseo, se inicia la paradoja de la expresión (MERLEAU PONTY, 1964, p. 144). [Traducción propia]

\section{CONSIDERACIONES FINALES}

Con todo, desde nuestro punto de vista, un objetivo central del $\mathrm{Cl}$ como técnica de danza podría ser el desarrollo de la movilidad y de la sensibilidad del conjunto del cuerpo a partir de las referencias de la mano y del ojo. Proponemos la denominación de "tacto extendido" a un tacto que partiendo de la piel de la mano se amplía hacia todo el cuerpo, desde los tejidos y pieles más internos hacia la piel exterior o piel espacial. Dicha extensión, apropiándonos de Gil (2006, p. 22), prolongaría el cuerpo incluso más allá de sus contornos visibles, sería piel convertida en espacio, un espacio que al bailar adquiere una gran diversidad de texturas. $\mathrm{Ni}$ la piel ni la mano son referentes exclusivos para el sentido del tacto; en la medida en que la piel se proyecta por espacio, se la podría considerar como un órgano entre cuyas funciones también está la vista.

La primera función del tacto en la danza $\mathrm{Cl}$ es el diálogo; a través del tacto se ven y se leen los cuerpos, se escucha y desarrolla la propuesta de movimiento conjunto; la segunda función es la puesta en juego de una amplia gama de técnicas derivadas de la cantidad de peso y de la cualidad de movimiento que se aplica, dependiendo de la dinámica que sucede entre las superficies de contacto; y en tercer lugar, hay que destacar la disponibilidad para el contacto, que se vincula a la habilidad para entregar o recibir peso, pero también para utilizar distintas partes del cuerpo de forma desinhibida. Determinar en profundidad las funciones de la vista durante la danza y en relación con el tacto es aún una tarea pendiente de la pedagogía del $\mathrm{Cl}$.

Por su parte, los análisis de Gibson (1986) presuponen la movilidad del observador, pero conservando la referencia estable del soporte del suelo y del cielo. El tipo de motricidad 
utilizada en sus experimentaciones incluye cambios posturales entre posiciones habituales (tumbado, sentado, de pie) y movimientos de locomoción como caminar o ir en bicicleta en los cuales se mantiene la posición erecta. La espacialidad esférica de la danza $\mathrm{Cl}$ - que utiliza todos los ejes y planos de movimiento-, exige utilizar todo el cuerpo (interno y externo, anterior, lateral o posterior) para sentir el espacio y a los otros cuerpos más allá de lo que nos impone nuestra visión frontal; es indispensable aprender a perder miedos para desplazarse hacia atrás y encontrar la forma de experimentar una danza multidireccional; ir hacia atrás tratando de sentir a través de la espalda, los miembros y los órganos internos es una tarea compleja en la que las funciones y los órganos de la visión y del tacto necesitan intercambiarse. Es preciso, por tanto, seguir cuestionándose el alcance, la organización y la didáctica de los sentidos.

Me pregunto cómo expandir las posibilidades y funciones del tacto y de la visión durante la danza; propongo, para ello la reversibilidad entre estos dos sentidos como la base para el diálogo corporal: la transferencia de operaciones de la mirada al tacto y viceversa. El juego con la gravedad y con la improvisación que caracteriza el $\mathrm{Cl}$ nos permite proyectarnos al espacio y llevar la experiencia de la danza a lugares inhabitados. Se trata de desplegar el tacto y la mirada a la vasta geografía del cuerpo, así como al dinámico paisaje que emerge entre los diferentes cuerpos en movimiento.

\section{REFERENCIAS}

ACKERMAN, Diane. Una historia natural de los sentidos. Barcelona: Anagrama, 1992.

AIKEN, Chris. Moulding/Draping. Contact Quarterly, v. 33, n. 2, p. 55, 2008.

ALESSI, Alito. Yes and Know Work. Contact Quarterly, v. 34, n. 2, p. 37, 2009.

BALES, Melanie; NETTL-FIOL, Rebecca. The body eclectic. Evolving Practices in Dance Training. Urbana \& Chicago: University of Illinois, 2008.

BANES, Sally. Terpsichore in sneakers: postmodern dance. Middleton, CT: Wesleyan University, 1987.

BAINBRIDGE-COHEN, Bonnie. Sensing, Feeling and Action. The Experiential Anatomy of Body-Mind Centering. Northampton: Contact Editions, 1993.

BENJAMIN, Adams. Making an Entrance: Theory and Practice for Disabled and Non-disabled Dancers. New York: Roudledge, 2002.

BERNARD, Michel. L'expressivité du corps. Quel Corps? n.7, p. 5-10, 1977.

BERNARD, Michel. De la création choréografique. Pantin: Centre National de la Danse, 2001.

BROOK, Annie. Awaking the Back Space. Contact Quarterly: Dance and Improvisation Journal, v. 41, n.1, p. 43, jan/jun. 2016.

BROZAS, María Paz. Danza, acrobacia y pedagogía corporal. In: CONGRESO DE LA ASOCIACIÓN ESPAÑOLA DE CIENCIAS DEL DEPORTE, 1. 2000. Libro de Actas....Cáceres: Universidad de Extremadura, 2000. v.1, p. 309-316. 
BROZAS, María Paz. El cuerpo en la evolución de la escritura de la danza contemporánea. Movimento, Porto alegre, v. 19, n. 3, p. 275-294, jul./set. 2013.

BROZAS, María Paz. Danza Contact Improvisation en la Universidad. Análisis de un proceso didáctico inclusivo. Tándem. Didáctica de la Educación Física, n. 51, p. 50-54, ene. 2016a.

BROZAS, María Paz. Expansión geográfica y conceptual de la danza Contact Improvisation en España (1990-2000). Retos, n. 30, p. 30-35, jul./sep. 2016b. Disponible en: <http://recyt.fecyt.es/ index.php/retos/article/view/36514/30087 >. Acceso en:9 ene.2017.

BUCKWALTER, Melinda. Composing While Dancing: an Improviser's Companion. Madison: University of Winconsin, 2011.

CLAVEL, Joanne; GINOT, Isabelle. Por uma Ecología da Somática? Estudos da Presença, v. 5, n.1, p. 85-100, jan./abr. 2015.

COOPER ALBRIGHT, Ann. Engaging bodies. The politics and poetics of corporeality. Middletown: Wesleyan University, 2013.

CHUNG, Ray. Listening. Contact Quarterly: Dance and Improvisation Journal, v. 31, n.1, p. 54, jan./jun. 2006.

CHRISTENSON, Neige. Modulation of Tone and Connection to Center. Contact Quarterly: Dance and Improvisation Journal, v. 38, n.1, p. 35, jan/jun. 2013.

DESPRÈS, Aurore. La relation pédagogiques dans le Contact Improvisation: le partage en mouvement. Nouvelles de Danse, n.46/47, p. 130-145, jui/aoû. 2001.

FONTAINE, Geisha. Les danses du temps. Pantin: Centre National de la Danse, 2004.

GAOS, José. La caricia. In: SERRANO, Agustín. Cuerpo vivido. Madrid: Encuentro, 2010. p. 53-85.

GIBSON, James. Observations on active touch. Psychological Review, v. 69, n.6, p. 477-491, 1962

GIBSON, James. The ecological approach to visual perception. London: Psychology Press, 2015.

GIL, José. A comunicação dos corpos: Steve Paxton. In: Movimento Total: o corpo e a dança. Lisboa: Relógio d’água, 2001. p. 131-156.

GIL, José. Paradoxical Body. TFR: The Drama Review, v. 50, n. 4, p. 21-35, 2006.

HOLMES, K.J. You walk into a room. Contact Quarterly: dance and improvisation journal, v. 37, n.1, p. 34, jan./jun. 2012.

HORRIGAN, Kristin. Slow-Motion Duet. Contact Quarterly: dance and improvisation journal, v. 39, n.1, p. 39, jan/jun. 2014.

HUGUES, Martin. Dance with the Body You Have. Contact Quarterly: dance and improvisation journal, v. 34, n. 1, p. 48-49, jan./jun. 2009.

KEOGHT, Martin. Falling. Contact Quarterly: dance and improvisation journal, v. 32, n. 2, p. 54, jul./dec. 2007. 
LEPKOFF, Daniel. An Ordinary Movement. Contact Quarterly: dance and improvisation journal v. 33, n. 1, p. 53, jan/jun. 2008.

LEITE, Fernanda. Contato Improvisação (Contact Improvisation): um diálogo em dança. Movimento, v.11, n.2, p. 89-110, maio/ago. 2005.

LIDELL, Lucy. El cuerpo sensual. Barcelona: Integral, 1989.

LITTLE, Nita. Place and Pour. Contact Quarterly: dance and improvisation journal, v. 32, n.1, p. 56, jan./jun. 2007.

LOUPPE, Laurence. Poétique de la danse contemporaine. Bruxelas: Contredanse, 2004.

LUZURIAGA, Lorenzo. Las Escuelas Nuevas. Madrid: Cosano, 1923.

MARTIN, Nina. The Fussy Dance: a low Ambition Entry into Contact Improvisation. Contact Quarterly: dance and improvisation journal, v. 35, n.1, p. 37, 2010.

MERLEAU PONTY, Maurice. Le visible et l'invisible. París: Gallimard, 1964. Disponible en: <https://monoskop.org/images/c/c6/Merleau Ponty Maurice Le Visible et L Invisible 19792001. pdf> . Acceso en: 9 ene. 2017.

MERLEAU PONTY, Maurice. Fenomenología de la percepción. Barcelona: Península, 1975.

MONTAGU, Ashey. Touching: the Human Significance of the Skin: New York: Columbia University, 1971.

MORRISSEY, Charlie. Room to move. Contact Quarterly: dance and improvisation journal, v. 36, n.1, p. 37, annual, 2011.

NELSON, Karen. Touch revolution: giving dance. In: STARK SMITH, Nancy. Contact Quarterly's Contact Improvisation sourcebook II: collected writings and graphics from Contact Quarterly dance journal 1993-2007. Northampton: Contact Editions, 2008. p. 103-104.

NELSON, Karen. Tones of Relaxation. Contact Quarterly: dance and improvisation journal, v. 31, n. 2, p. 61, jul./dec. 2006.

NELSON, Lisa. Á travers vos yeux. Nouvelles de Danse, n.48/49, p. 9-26, sep./mar. 2001.

PAXTON, Steve. Contact Improvisation. The Drama Review, v. 19, n. 1, p. 40-42, mar. 1975.

PAXTON, Steve. Drafting Interior Techniques. Contact Quarterly: dance and improvisation journal, v. 18, n. 1, p. 64-78, jan./jun.1993.

PAXTON, Steve. To Touch. Contact Quarterly: dance and improvisation journal, v. 21, n. 2 , p. 50-51, jul./dec. 1996.

PAXTON, Steve; BIGÉ, Romain. Mouvements ancestraux. Le Contact Improvisation et les premiers contacts. Repères, cahier de danse, v. 36, p. 3-6, nov. 2015.

PAXTON, Steve; KILCOYNE, Anne; MOUNT, Kate. On the Braille in the Body: An Account of the Touchdown Dance Integrated Workshops with the Visually Impaired and the Sighted. Dance Research: The Journal of the Society for Dance Research, v. 11, n. 1, p. 3-51, mar./jun. 1993. 
PEETERS, Jeroen. Through the Back. Situating Vision between Moving Bodies. Helsinki: University of the arts Helsinki Theatre Academy, 2015.

PICARD, Dominique. Del código al deseo. Buenos Aires: Paidós, 1986.

ROBIN, Gilmore. Learning to Touch: Contact and Alexander Training in Japan. In: Contact Quarterly's Contact Improvisation sourcebook II: collected writings and graphics from Contact Quarterly dance journal 1993-2007. Northampton: Contact Editions, 2008. p. 75-76.

SUNGHE PAEK, Margaret. Beginning, Approaching, Practicing, Dancing. Contact Quarterly: dance and improvisation journal, v. 40, n.1, p. 36, jan./jun. 2015.

SUQUET, Annie. Escenas. El cuerpo danzante: un laboratorio de la percepción. In: Historia del cuerpo en el siglo XX. Madrid: Taurus, 2006. v.3, p. 379-400.

TAMPINI, Marina. El uso de los sentidos. In: Cuerpos e ideas en danza. Una mirada sobre el Contact Improvisation. Buenos Aires: Instituto Universitario Nacional del Arte, 2012. p. 53-61.

TORRENTS, Carlota; CASTAÑER, Marta. Educación integral mediante el Contact Improvisation. Tándem. Didáctica de la Educación Física, n. 26, p. 91-100, ene./mar. 2008.

TORRENTS, Carlota et al. Discovering new ways of moving: observational analysis of motor creativity while dancing contact improvisation and the influence of the partner. Journal of Creative Behavior, v. 44, n. 1, p. 45-61, 2010.

VAYER, Pierre. El diálogo corporal. Madrid: Científico-Médica, 1977.

USKI, Isabelle. Nature et enjeux du toucher dans la pratique de Contact Improvisation. 2003.108f. Thèse (Maistrise) - Département de Danse, Université Paris 8, Paris, 2003. 
